\title{
Interindividual Variation in Fornix Microstructure and Macrostructure Is Related to Visual Discrimination Accuracy for Scenes But Not Faces
}

\author{
Mark Postans, ${ }^{1,2,3 *}$ Carl J. Hodgetts, ${ }^{1,2,3 *}$ Matthew E. Mundy, ${ }^{4}$ Derek K. Jones, ${ }^{1,2,3}$ Andrew D. Lawrence, ${ }^{1,2,3} \dagger$ \\ and Kim S. Graham ${ }^{1,2,3 \dagger}$ \\ ${ }^{1}$ Wales Institute of Cognitive Neuroscience, ${ }^{2}$ Cardiff University Brain Research Imaging Centre, School of Psychology, and ${ }^{3}$ Neuroscience and Mental Health \\ Research Institute, Cardiff University, CF10 3AT, United Kingdom, and ${ }^{4}$ School of Psychology and Psychiatry, Monash University, Clayton, Victoria 3800, Australia
}

Transection of the nonhuman primate fornix has been shown to impair learning of configurations of spatial features and object-in-scene memory. Although damage to the human fornix also results in memory impairment, it is not known whether there is a preferential involvement of this white-matter tract in spatial learning, as implied by animal studies. Diffusion-weighted MR images were obtained from healthy participants who had completed versions of a task in which they made rapid same/different discriminations to two categories of highly visually similar stimuli: (1) virtual reality scene pairs; and (2) face pairs. Diffusion-MRI measures of white-matter microstructure [fractional anisotropy (FA) and mean diffusivity (MD)] and macrostructure (tissue volume fraction, $f$ ) were then extracted from the fornix of each participant, which had been reconstructed using a deterministic tractography protocol. Fornix MD and $f$ measures correlated with scene, but not face, discrimination accuracy in both discrimination tasks. A complementary voxelwise analysis using tract-based spatial statistics suggested the crus of the fornix as a focus for this relationship. These findings extend previous reports of spatial learning impairments after fornix transection in nonhuman primates, critically highlighting the fornix as a source of interindividual variation in scene discrimination in humans.

Key words: discrimination learning; fornix; hippocampus; scene discrimination; tractography

\section{Introduction}

The fornix is a prominent white-matter tract linking the hippocampus to several subcortical (e.g., anterior thalamic nuclei, mammillary bodies) and cortical (e.g., prefrontal cortex) regions (Saunders and Aggleton, 2007). Given that these areas are important for successful learning and memory (Aggleton, 2008), their ability to communicate with one another via the fornix may also be critical for performance in tasks challenging these cognitive domains.

Indeed, fornix transection produces deficits in memory tasks

Received Jan. 3, 2014; revised July 7, 2014; accepted July 11, 2014.

Author contributions: M.P., C.J.H., M.E.M., D.K.J., and K.S.G. designed research; M.P. and C.J.H. performed research; M.P., C.J.H., and A.D.L. analyzed data; M.P., C.J.H., M.E.M., D.J., A.D.L., and K.S.G. wrote the paper.

This research was funded by Biotechnology and Biological Sciences Research Council Grant BB/1007091/1 and Medical Research Council Grant G1002149. We thank Nils Muhlert and colleagues at the Cardiff University Brain Research Imaging Centre for help and support with imaging data collection and analysis. We also thank Ofer Pasternak and Greg Parker for implementing the free-water correction pipeline that was used during our DWI preprocessing.

${ }^{*}$ M.P. and C.J.H. are joint first authors.

†A.D.L. and K.S.G. are joint senior authors.

The authors declare no competing financial interests.

This article is freely available online through the J Neurosci Author Open Choice option.

Correspondence should be addressed to Mark Postans, Wales Institute of Cognitive Neuroscience, School of

Psychology, Cardiff University, Cardiff, CF10 3AT. E-mail: PostansM@cardiff.ac.uk.

DOI:10.1523/JNEUROSCI.0026-14.2014

Copyright (c) 2014 Postans et al.

This is an Open Access article distributed under the terms of the Creative Commons Attribution License (http://creativecommons.org/licenses/by/3.0), which permits unrestricted use, distribution and reproduction in any medium provided that the original work is properly attributed. that are also sensitive to hippocampal lesions (e.g., object-in-scene memory tasks), consistent with the proposal that the fornix is one component of an extended hippocampus-fornix-mammillary body-anterior thalamic network involved in the representation of complex visual scenes and the arrangement of objects within those scenes (Gaffan, 1994). More recently, Buckley et al. (2004) demonstrated that, after fornix transection, macaque monkeys showed impaired concurrent visual discrimination learning between highly similar "tadpole" stimuli individually defined by unique spatial features. However, the extent to which the fornix is specialized for spatial memory is unclear, because in some contexts fornix transection can impair object discrimination learning (Wilson et al., 2007) and object novelty detection (Hunsaker and Kesner, 2009).

Studies in humans with fornix damage also highlight a key role in memory but have tended to focus on dissociations between memory processes (e.g., recollection vs familiarity; Vann et al., 2008). To date, there has been little investigation of the preferential contributions of the human fornix to spatial versus nonspatial learning and memory, in particular using tasks similar to those developed in animal studies (Bussey et al., 2002; Buckley et al., 2004).

Here, we asked healthy young participants to perform two tasks in which they were required to make same/different judgments to pairs of highly visually similar scenes versus pairs of highly similar faces. Analogous discrimination tasks are sensitive to medial temporal lobe (MTL) lesions in nonhuman primates (Bussey et al., 2002) and humans (Barense et al., 2005; Mundy et 
al., 2013). Both deterministic white-matter tractography and tract-based spatial statistics (TBSS) approaches were applied to obtain diffusion-MRI indices of fornix microstructure [fractional anisotropy (FA) and mean diffusivity (MD)] and macrostructure (tissue volume fraction, $f$ ) in our participants. FA and MD are influenced by microstructural properties, including myelination and axon density (Beaulieu, 2002), and variation in these measures within the fornix has been shown to correlate with memory performance in older individuals (Metzler-Baddeley et al., 2011). $f$ indicates the fraction of the diffusion-weighted MR signal attenuation that is attributable to diffusion occurring in tissue rather than in free water (Metzler-Baddeley et al., 2012) and can be used to describe the proportion of a tract reconstruction that is composed of tissue (i.e., its relative tissue volume). If the "extended hippocampal network" (Gaffan, 1994) is especially critical for spatial discrimination learning, we predicted that interindividual variation in fornix microstructural and macrostructural measures would be associated with participants' overall scene, but not face, discrimination accuracy.

\section{Materials and Methods}

Participants. Diffusion-weighted MRI and behavioral data were collected from 27 healthy participants. All participants provided written informed consent for participation in the study, which was approved by the Cardiff University School of Psychology Research Ethics Committee.

Analyses are reported on two tasks separately, because not every participant completed both tasks ( 16 completed both). Task A included 21 participants (two males; aged 18-22 years; mean \pm SD, $19.0 \pm 1.1$ ), and Task B included 22 participants (two males; aged 19-23 years; mean \pm SD, $19.9 \pm 1.0$ ).

Tasks. The two tasks involved making sequential discriminations between pairs of highly similar scenes and faces (Mundy et al., 2013; see example stimuli in Fig. $1 A$ ). In Task A, which was administered on the same day that diffusion-weighted images (DWIs) were acquired, participants made rapid same/different discrimination judgments to eight unique pairs of highly similar faces and scenes. For each visual category, 96 discrimination trials (Fig. 1B) were distributed over two task runs. In each run, four pairs were repeated over $\mathrm{ABCD}$ blocks until participants had made 48 discriminations. Consistent with Mundy et al. (2013), participants were preexposed to half of the pairs from each category. The preexposed pairs were the same for all participants.

In Task $B$, which was administered $\sim 10$ months later, participants made rapid discrimination judgments to four pairs of highly similar faces, scenes, and dot patterns over ABCD blocks (total of 64 discriminations per category). Participants were preexposed to half of the pairs from each category, and the preexposed pairs were counterbalanced across participants. Three of the four pairs of both the face and scene stimuli were taken from Task A. Because the dot-pattern condition is not relevant to the current hypotheses, we do not discuss this further.

MRI acquisition. Whole-brain diffusion-weighted MRI data were acquired using a 3 T GE HDx Signa scanner with an eight-channel head coil at the Cardiff University Brain Research Imaging Centre. Images were acquired using a diffusion-weighted single-shot spin-echo echo-planar imaging pulse sequence $\left(\mathrm{TE}=87 \mathrm{~ms}\right.$; field of view, $23 \times 23 \mathrm{~cm}^{2}$; $96 \times 96$ acquisition matrix; 60 contiguous slices acquired along an oblique-axial plane with $2.4 \mathrm{~mm}$ thickness and no gap), and the scans were cardiacgated using a peripheral pulse oximeter. Gradients were applied along 30 isotropically distributed orientations with $b=1200 \mathrm{~s} / \mathrm{mm}^{2}$ (Jones et al., 1999). Three non-DWIs with $b=0 \mathrm{~s} / \mathrm{mm}^{2}$ were also acquired.

DWI preprocessing. DWI preprocessing was performed with ExploreDTI (Leemans et al., 2009). We also used custom MATLAB scripts to perform a free-water-elimination procedure that applies post hoc voxelwise correction for partial volume errors attributable to free-water contamination in diffusion indices before individual-subject tractography (Pasternak et al., 2009). This process also produces voxelwise maps of the tissue volume fraction $f$. These images were later intersected with the results of our individual-subject tractography protocol (below) to extract $f$ from the reconstructions.

Tractography protocol. Tractography was performed from all voxels in participants' (free-water-corrected) native diffusion space in ExploreDTI using a deterministic tractography algorithm based on constrained spherical deconvolution (Tournier et al., 2008; Jeurissen et al., 2011). We used a step size of $1 \mathrm{~mm}$ and an angle threshold of $30^{\circ}$ to prevent the reconstruction of anatomically implausible fibers. A multiple region-of-interest (ROI) approach was then used to isolate the fornix bilaterally (mean \pm SD voxel count, $1227.5 \pm 406.7$; range, $460-1931$ ) from the results of this whole-brain tractography procedure (Metzler-Baddeley et al., 2011). Mean $\mathrm{FA}, \mathrm{MD}$, and $f$ indices were subsequently extracted from the reconstructions in ExploreDTI (Jones et al., 2005).

To test the specificity of any significant correlations between fornix FA/MD/f and face/scene discrimination accuracy, we also obtained mean $\mathrm{FA} / \mathrm{MD} / f$ indices from the main occipito-temporal associative whitematter pathway: the inferior longitudinal fasciculus (ILF; Catani et al., 2003). The right and left ILF were reconstructed (mean \pm SD voxel count, $2910.2 \pm 788.1$; range, $1628-4655$ ) using a two-ROI approach (Wakana et al., 2007), and for comparability with the fornix, the average of the measures obtained from the two hemispheres was calculated to create a single value per participant.

Two-tailed Pearson's correlation statistics were calculated to establish whether fornix/ILF FA, MD, or $f$ were related to overall discrimination accuracy for scene or face pairs in either Task A or B. A Bonferroniadjusted significance threshold of $p \leq 0.05$ was adopted (comparison of each microstructure/macrostructure metric with two behavioral variables per task; required $p \leq 0.025)$. All correlations are reported with $95 \%$ confidence intervals (CIs) around the effect size estimates, based on 1000 bootstrapped samples of the data. Directional $t$ tests for dependent correlations were used to determine significant differences between correlations.

TBSS approach. We also conducted a complementary TBSS analysis with threshold-free cluster enhancement (Smith et al., 2006; Smith and Nichols, 2009). Our analyses were initially restricted to the fornix using a suitable binary mask. This was created by thresholding (20\%) and binarizing a probabilistic mask of the fornix from the Juelich Histological Atlas in FSL (FMRIB Software Library). This image was then intersected with the skeletonized mean FA image of all participants (itself thresholded at 0.2 ) separately for Tasks A and B. A thresholded (30\%) and binarized mask of the corpus callosum (also provided with the Juelich Histological Atlas in FSL) was then subtracted from this intersected fornix mask to ensure that it did not contain any voxels that actually corresponded to this larger white-matter structure. This procedure resulted in a binary fornix mask for both Task A ( 1240 voxels at $1 \times 1 \times 1 \mathrm{~mm})$ and Task B (1158 voxels at $1 \times 1 \times 1 \mathrm{~mm})$. General linear models and contrasts of interest were then implemented to identify regions within this mask in which a given diffusion-MRI metric was more predictive of 
Table 1. Mean \pm SD and performance range on Tasks $A$ and $B$ separated by condition (scenes and faces)

\begin{tabular}{lcl}
\hline Condition & Mean \pm SD & Range \\
\hline A Scenes & $74.5 \pm 11.85$ & \\
\multicolumn{1}{l}{ Faces } & $72.12 \pm 5.55$ & $53.13-90.63$ \\
B & $79.69 \pm 12.57$ & $63.54-80.21$ \\
$\quad$ Scenes & $70.03 \pm 11.04$ & $45.31-95.31$ \\
Faces & & $43.75-87.5$ \\
\hline
\end{tabular}

Table 2. Mean \pm SD FA, MD $\left(\times 10^{-3} \mathrm{~mm}^{2} \mathrm{~s}^{-1}\right)$, and $f$ in both the fornix and the ILF of all 27 participants

\begin{tabular}{llll}
\hline Tract & FA & MD & $f$ \\
\hline Fornix & $0.373 \pm 0.032$ & $1.056 \pm 0.055$ & $0.727 \pm 0.035$ \\
ILF & $0.428 \pm 0.023$ & $0.757 \pm 0.017$ & $0.896 \pm 0.013$ \\
\hline
\end{tabular}

scene versus face discrimination accuracy (and vice versa). We adopted a threshold of $p \leq 0.005$ uncorrected, cluster size $\geq 3$ voxels for this analysis, which yielded a corrected threshold of $p \leq 0.05$, determined by Monte Carlo simulation using AFNI (Automated Functional NeuroImaging) AlphaSim (http://afni.nimh.nih.gov). A whole-brain TBSS analysis was also performed; we adopted a more stringent threshold of $p \leq 0.001$ uncorrected, cluster size $\geq 10$ voxels, as reported by Rudebeck et al. (2009), to explore structure-function relationships in the rest of the white-matter skeleton (separately for Tasks A and B). To control for potential false positives in the whole-brain results, we report only those regions that showed a consistent pattern across Tasks A and B. All reported coordinates are in MNI 152 space.

\section{Results}

Table 1 describes participants' performance in both tasks; analyses of this data are reported, separately by task, below. Table 2 shows the mean $\pm \mathrm{SD}$ of values obtained for $\mathrm{FA}, \mathrm{MD}$, and $f$ in the fornix and ILF. Before reporting the analyses testing our key hypotheses, we assessed the relationship between $\mathrm{FA}, \mathrm{MD}$, and $f$ by calculating correlation coefficients between each measure for each tract for all 27 participants. Fornix FA was strongly correlated with both fornix MD and $f(r=-0.760, p<0.001$, $95 \% \mathrm{CI}=-0.917,-0.487 ; r=0.685, p<0.001,95 \% \mathrm{CI}=0.419$, 0.851 , respectively). Fornix MD and $f$ were also highly correlated $(r=-0.795, p<0.001,95 \% \mathrm{CI}=-0.906,-0.603)$. Turning to the ILF, MD and $f$ were highly correlated $(r=-0.969, p<0.001$, $95 \% \mathrm{CI}=-0.986,-0.932)$. ILF FA was correlated with ILF MD and $f(r=-0.275, p=0.166,95 \% \mathrm{CI}=-0.618,0.169 ; r=0.338$, $p=0.085,95 \% \mathrm{CI}=-0.100,0.661)$, but these correlations failed to reach statistical significance.

\section{Task A: behavioral data}

After dividing the discrimination test trials into four discrete time bins (Mundy et al., 2013), a 2 (exposure: preexposed vs novel) $\times$ 2 (category: scenes vs faces) $\times 4$ (bin number: $1,2,3$, and 4 ) repeated-measures ANOVA revealed no significant main effects of bin number $\left(F_{(2,60)}=1.585, p=0.202\right)$ or category $\left(F_{(1,20)}=\right.$ $1.147, p=0.297)$ on performance in Task A. There was a trend toward a main effect of exposure because discrimination accuracy was numerically greater for novel relative to preexposed pairs $\left(F_{(1,20)}=3.965, p=0.06\right)$. There was a significant category $\times$ bin number interaction $\left(F_{(3,60)}=7.45, p<0.001\right)$, which post hoc pairwise comparisons (Bonferroni's-corrected) revealed to be driven by higher scene discrimination accuracy in bins 2 and 3 relative to bin 1 ( $p=0.012$ and 0.002 , respectively). Scene discrimination accuracy in bin 4 was nonsignificantly different from that obtained in bins 1,2 , or 3 ( $p=0.135,1.0$, and 1.0, respectively). Face-pair discrimination accuracy did not differ across any of the four bins (all p's $>0.773$ ). These findings indicate that overall discrimination accuracy is similar across the two visual categories and that there was no significant and enduring improvement in discrimination accuracy from repetition or exposure to half of the stimuli before the discrimination test. This was true for both categories of stimuli.

\section{Task A: tractography}

Fornix $f$ correlated significantly with overall discrimination accuracy for scenes $(r=0.597, p=0.004,95 \% \mathrm{CI}=0.174,0.791)$ but not faces $(r=0.278, p=0.223,95 \% \mathrm{CI}=-0.222,0.660)$, and these correlations were significantly different $\left(t_{(18)}=1.69, p=\right.$ 0.054; Fig. 2A). Likewise, fornix MD correlated significantly with discrimination accuracy for scenes $(r=-0.729, p<0.001$, $95 \% \mathrm{CI}=-0.908,-0.359)$ but not faces $(r=-0.138, p=0.552$, $95 \% \mathrm{CI}=-0.515,0.319)$, and these correlations were also significantly different $\left(t_{(18)}=3.986, p<0.001\right.$; Fig. $\left.2 B\right)$. There was also a significant correlation between fornix FA and discrimination accuracy for scenes $(r=0.545, p=0.011,95 \% \mathrm{CI}=0.178$, $0.816)$ but not faces $(r=0.282, p=0.215,95 \% \mathrm{CI}=-0.082$, $0.592)$. The difference between these correlations did not reach significance $\left(t_{(18)}=1.34, p=0.099\right.$; Fig. $\left.2 C\right)$.

With regard to the ILF, there were no Bonferroni-adjusted significant correlations between discrimination performance and either microstructural or macrostructural measures.

\section{Task B: behavioral data}

There was a significant main effect of category on discrimination accuracy in Task B, which reflected higher performance for scenes than faces $\left(F_{(1,21)}=17.153, p<0.001\right)$. There was no main effect of bin number, but the main effect of exposure was significant, with discrimination accuracy being higher for preexposed pairs relative to those that were novel at test $\left(F_{(1,21)}=6.621, p=0.018\right)$. There was also a significant category $\times$ exposure interaction, which post hoc tests revealed reflected better discrimination accuracy for preexposed compared with novel scenes $(p=0.005)$ but not faces $(p=$ 0.483 ). All other interactions were nonsignificant. In brief, although there was no significant improvement in discrimination accuracy over the task, overall scene/pair discrimination accuracy did benefit from exposure to stimuli before the main task.

\section{Task B: tractography}

As in Task A, fornix $f$ correlated significantly with discrimination accuracy for scenes $(r=0.609, p=0.003,95 \% \mathrm{CI}=0.397,0.857)$ but not faces $(r=0.235, p=0.292,95 \% \mathrm{CI}=-0.158,0.608)$, and these correlations were significantly different $\left(t_{(19)}=2.26\right.$, $p=0.018$; Fig. $2 D$ ). Likewise, fornix MD correlated significantly with discrimination accuracy for scenes $(r=-0.550, p=0.008$, $95 \% \mathrm{CI}=-0.785,-0.205)$ but not faces $(r=-0.266, p=0.231$, $95 \% \mathrm{CI}=-0.574,0.157)$, and the difference between these correlations showed a strong trend toward significance $\left(t_{(19)}=1.61\right.$, $p=0.062$; Fig. $2 E$ ). There was also a marginally significant correlation between fornix $\mathrm{FA}$ and discrimination accuracy for scenes $(r=0.468, p=0.028,95 \% \mathrm{CI}=0.180,0.759)$ but not faces $(r=0.263, p=0.237,95 \% \mathrm{CI}=-0.111,0.632)$; the difference between these correlations was not significant $\left(_{(19)}=1.10, p=\right.$ 0.144; Fig. 2F).

Our ILF analyses revealed a significant correlation between ILF FA and overall face discrimination accuracy $(r=0.477, p=$ $0.025,95 \% \mathrm{CI}=0.008,0.823)$ but not scene discrimination performance $(r=0.170, p=0.449,95 \% \mathrm{CI}=-0.350,0.547)$, and the difference between these two correlations showed a strong 
Fornix $f$
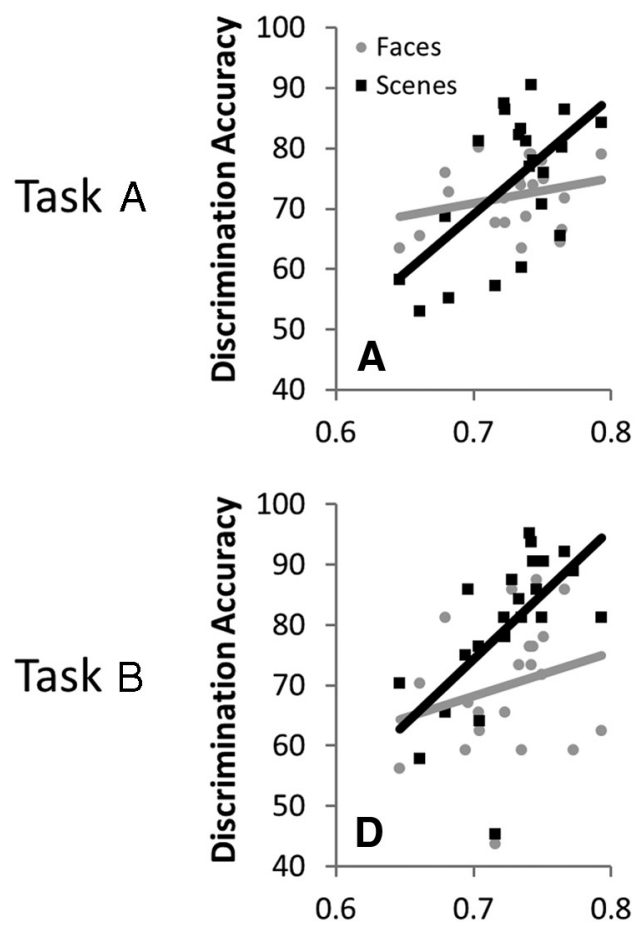

Fornix MD
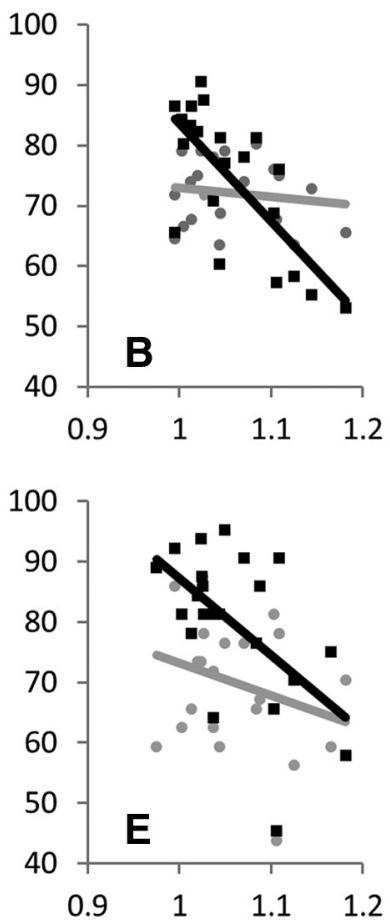

Fornix FA
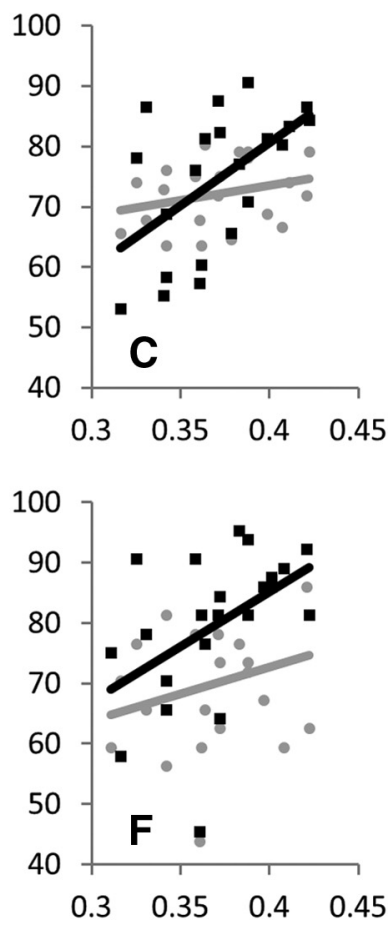

Figure 2. Relationship between face/scene discrimination accuracy (gray/black lines, respectively) and fornix $f, M D\left(\times 10^{-3} \mathrm{~mm}^{2} \mathrm{~s}^{-1}\right)$ and FA in Task $A(\boldsymbol{A}, \boldsymbol{B}, \boldsymbol{C}$, respectively) and Task B $(\boldsymbol{D}, \boldsymbol{E}$, $\boldsymbol{F}$, respectively).

trend toward significance $\left(t_{(19)}=1.67, p=0.056\right)$. All other ILF statistical tests were nonsignificant.

Given the intercorrelations between FA, MD, and $f$ (see beginning of Results), $z$-scores for each participant's FA, $f$, and $1-\mathrm{MD}$ estimates from the fornix and the ILF (separately for Tasks A and B) were calculated. These $z$-scores were averaged to create a composite $z$-score (CZ) for each tract, separately for each individual; this was then correlated with scene and face discrimination performance. In both Tasks A and B, we found that fornix CZ correlated with discrimination accuracy for scenes $(r=0.683, p=$ $0.001,95 \% \mathrm{CI}=0.349,0.858 ; r=0.600, p=0.003,95 \% \mathrm{CI}=$ $0.383,0.813$, respectively) but not faces $(r=0.255, p=0.265$, $95 \% \mathrm{CI}=-0.232,0.622 ; r=0.282, p=0.204,95 \% \mathrm{CI}=-0.100$, 0.661 , respectively), and these correlations were significantly different $\left(t_{(18)}=2.51, p=0.011 ; t_{(19)}=1.88, p=0.0375\right.$, respectively). In comparison, all corresponding statistical tests in the ILF were nonsignificant.

\section{TBSS approach}

Defining the fornix as the ROI in TBSS, we found confirmatory evidence of a crucial role for the fornix in scene, but not face, discrimination. In Task A, there was a cluster of 16 voxels in the right crus of the fornix (maximum $t=4.12, x=12, y=-28, z=$ 12; Fig. $3 A$ ), in which MD was more predictive of scene than face accuracy. In Task B, there were two clusters each containing 4 voxels in which MD was more predictive of scene than face accuracy: (1) cluster 1 was located in the right crus of the fornix (maximum $t=2.07, x=14, y=-29, z=13$; Fig. $3 B$ ); (2) cluster 2 was located in the left crus of the fornix (maximum $t=4.92, x=$ $-18, y=-38, z=7)$. In both tasks, there were no clusters in which FA or $f$ was more predictive of scene versus face discrimination accuracy. The reverse contrast revealed no clusters in the

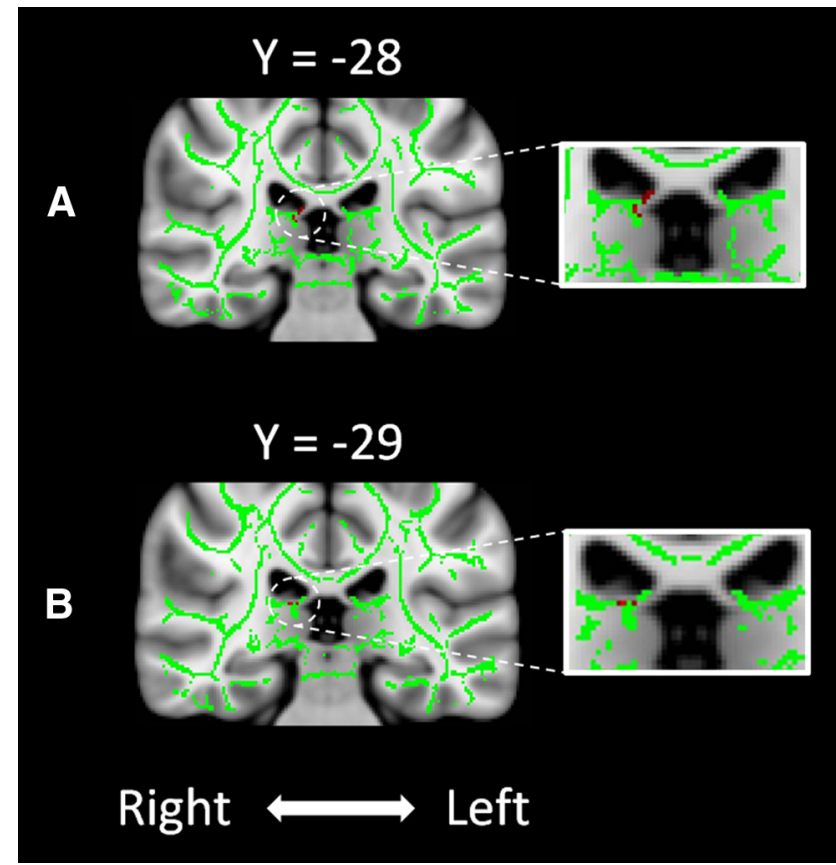

Figure 3. Clusters identified in the fornix-specific TBSS analysis (red voxels), in which MD was more predictive of discrimination accuracy for scenes than faces, in Tasks $A(\boldsymbol{A})$ and $B(\boldsymbol{B})$. The clusters are overlaid on coronal sections of the MNI template and the white matter skeleton (green voxels) of all participants, separately for Tasks A and B.

fornix in which a diffusion-MRI metric was more predictive of discrimination accuracy for faces than scenes.

In the whole-brain TBSS analysis, for MD and $f$, there were no regions outside the fornix that predicted scene discrimination 
accuracy to a greater extent than faces (and vice versa). For FA, there were clusters in the white matter of the cerebellum, in which FA was more predictive of discrimination accuracy for scenes than faces (Task A: a single cluster of 12 voxels in the white matter of the right cerebellum, maximum $t=2.27, x=34, y=-52, z=$ -43 ; Task B: three clusters in the right cerebellum, cluster 1: $n=$ 11 voxels, maximum $t=3.17, x=26, y=-63, z=-22$; cluster $2: n=11$ voxels, maximum $t=2.35, x=4, y=-59, z=-32$; cluster 3: $n=10$ voxels, maximum $t=3.74, x=38, y=-58, z=$ $-36)$. The category-sensitive structure-function associations that were identified in the fornix-specific TBSS analyses did not survive correction at the whole-brain level.

\section{Discussion}

Our study revealed that interindividual variation in fornix microstructure and macrostructure was related to visual discrimination accuracy for scenes, but not faces, across two perceptual discrimination tasks. Importantly, this effect was not evident in another whitematter bundle (ILF), implying specificity in the association between scene discrimination ability and fornix macrostructure and microstructure. There was also evidence (based on deterministic tractography) that ILF FA was associated with discrimination accuracy for faces, and not scenes, in Task B, and an exploratory TBSS analysis further revealed a significant correlation between FA in cerebellar white matter and overall scene discrimination accuracy.

By demonstrating selective structure-function associations in the healthy intact brain, our findings extend conclusions from lesion studies that (1) hippocampal damage in humans impairs scene-pair discrimination learning while leaving face-pair discrimination learning intact (Mundy et al., 2013), and (2) fornix transection in nonhuman primates impairs the ability to learn concurrent visual discriminations between spatial stimuli (Buckley et al., 2004). The results confirm that the fornix is a key part of an extended hippocampal circuit involved in the representation of scene stimuli (Gaffan, 1994). More recent accounts have extended the theory by Gaffan (1994) by proposing that the hippocampus and perirhinal cortex support complex representations of spatial and nonspatial visual feature conjunctions, respectively (Graham et al., 2010; Saksida and Bussey, 2010). According to these views, the hippocampus is critical for scene memory but also perception, whereas the perirhinal cortex supports memory and perception for individual objects and faces. Lee et al. (2005) found that patients with hippocampal lesions were selectively impaired, relative to controls, in their ability to discriminate highly visually similar scenes (Aly et al., 2013). In contrast, patients with more extensive MTL damage that included the hippocampus and perirhinal cortex presented with additional discrimination impairments for faces and objects (Lee et al., 2005; Barense et al., 2007).

With their emphasis on the distinct visual representations supported by the hippocampus and perirhinal cortex, these "representational" models differ from MTL accounts focusing on distinct memory processes (Aggleton and Brown, 1999). Although representation-based views can readily accommodate the findings reported here, process-based accounts make limited predictions about how particular stimulus categories will differentially engage the hippocampal network. Therefore, representation-based MTL perspectives may offer new insights into the complex role of hippocampal white-matter connections in human cognition, including evidence that fornix microstructure is more strongly related to recollection-based recognition memory for scenes than objects (Rudebeck et al., 2009). In this context, mean ILF FA was associated with discrimination accuracy in Task B for faces but not scenes. Although this finding needs to be considered with caution, represen- tational accounts may also provide a useful framework for understanding how the ILF, as the major white-matter tract traversing the visual ventral stream (Catani et al., 2003), contributes to typical and atypical face processing (Thomas et al., 2009).

Representation-based accounts suggest that MTL representations may have both perceptual and mnemonic applications, and our findings could be interpreted as supporting this view. We were unable to detect any enduring improvement in discrimination accuracy over the course of either Task A or B, and there was no effect of previous exposure on overall discrimination accuracy in Task A. Particularly with Task A then, the behavioral data indicates that mnemonic processes were not necessarily recruited to support discrimination judgments. Notably, however, the stimuli used here were not "trial-unique," and although there was no statistically robust learning, it is possible that memory processes could have supported participants' discrimination judgments over time (Kim et al., 2011).

One advantage of a tract reconstruction approach is that it may be more sensitive to subtle long-range differences in whitematter microstructure compared with voxel-based DTI methods (Keedwell et al., 2012). Furthermore, by constraining our analyses to a small number of ROIs by using a hypothesis-driven approach, we reduce the risk of reporting (1) false-positive effects in regions for which we have no specific predictions and (2) falsenegative effects when true structure-function relationships are obscured because of corrections for large numbers of statistical comparisons. However, the complementary TBSS voxelwise analysis did show that the fornix MD-scene discrimination association was strongest in the crus of the fornix. In addition, wholebrain TBSS revealed clusters within cerebellar white matter in which FA was more associated with scene than face discrimination. Notably, many cerebellar subregions show correlated activity with the hippocampus at rest (Sang et al., 2012), and cerebellarhippocampal interactions may contribute to spatial navigation (Rochefort et al., 2013).

Although fornix MD correlated with scene-pair discrimination accuracy, fornix FA was less strongly associated with performance. This finding could reflect the greater sensitivity of the fornix MD to experience-dependent changes (Lebel et al., 2008; Hofstetter et al., 2013). Because FA and MD are both affected by multiple axonal properties, including myelination, density, diameter, and configuration, it is not yet possible to attribute differences between our FA/MD findings to a single white-matter subcomponent (Jones et al., 2013). That fornix $f$ was also associated with successful scene discrimination accuracy, as well as $\mathrm{MD}$, additionally highlights a contribution of fornix macrostructure to variation in scene discrimination. Given the intercorrelations between these imaging metrics and that a fornix CZ score was strongly correlated with scene, but not face, discrimination learning in the fornix, our findings imply that individual variation in some specific property of the fornix, to which multiple diffusion-MRI metrics are sensitive, is predictive of scene discrimination accuracy. However, it is also possible that interindividual variation in both the macrostructural and microstructural properties of the fornix contribute to performance in our tasks (Lebel and Beaulieu, 2011; Loui et al., 2011; Saygin et al., 2013).

In summary, we found that interindividual variation in fornix microstructure and macrostructure in healthy participants was related to discrimination accuracy for pairs of visually similar scenes but not faces. This finding augments lesion studies in which hippocampal damage results in selective impairments in spatial discrimination learning (Graham et al., 2006; Mundy et al., 2013) and highlights a critical role for the human fornix in 
high-level spatial, but not face, discrimination, consistent with representational accounts (Graham et al., 2010).

\section{References}

Aggleton JP (2008) EPS mid-career award 2006. Understanding anterograde amnesia: disconnections and hidden lesions. Q J Exp Psychol 61: 1441-1471. CrossRef Medline

Aggleton JP, Brown MW (1999) Episodic memory, amnesia, and the hippocampal-anterior thalamic axis. Behav Brain Sci 22:425-444, discussion 444-489. Medline

Aly M, Ranganath C, Yonelinas AP (2013) Detecting changes in scenes: the hippocampus is critical for strength-based perception. Neuron 78:11271137. CrossRef Medline

Barense MD, Bussey TJ, Lee ACH, Rogers TT, Davies RR, Saksida LM, Murray EA, Graham KS (2005) Functional specialization in the human medial temporal lobe. J Neurosci 25:10239-10246. CrossRef Medline

Barense MD, Gaffan D, Graham KS (2007) The medial temporal lobe processes online representations of complex objects. Neuropsychologia 45: 2963-2974. CrossRef Medline

Beaulieu C (2002) The basis of anisotropic water diffusion in the nervous system - a technical review. NMR Biomed 15:435-455. CrossRef Medline

Buckley MJ, Charles DP, Browning PGF, Gaffan D (2004) Learning and retrieval of concurrently presented spatial discrimination tasks: role of the fornix. Behav Neurosci 118:138-149. CrossRef Medline

Bussey TJ, Saksida LM, Murray EA (2002) Perirhinal cortex resolves feature ambiguity in complex visual discriminations. Eur J Neurosci 15:365-374. CrossRef Medline

Catani M, Jones DK, Donato R, Ffytche DH (2003) Occipito-temporal connections in the human brain. Brain 126:2093-2107. CrossRef Medline

Gaffan D (1994) Scene-specific memory for objects: a model of episodic memory impairment in monkeys with fornix transaction. J Cogn Neurosci 6:305-320. CrossRef Medline

Graham KS, Scahill VL, Hornberger M, Barense MD, Lee ACH, Bussey TJ, Saksida LM (2006) Abnormal categorization and perceptual learning in patients with hippocampal damage. J Neurosci 26:7547-7554. CrossRef Medline

Graham KS, Barense MD, Lee ACH (2010) Going beyond LTM in the MTL: A synthesis of neuropsychological and neuroimaging findings on the role of the medial temporal lobe in memory and perception. Neuropsychologia 48:831-853. CrossRef Medline

Hofstetter S, Tavor I, Tzur Moryosef S, Assaf Y (2013) Short-term learning induces white matter plasticity in the fornix. J Neurosci 33:12844-12850. CrossRef Medline

Hunsaker MR, Kesner RP (2009) Transecting the dorsal fornix results in novelty detection but not temporal ordering deficits in rats. Behav Brain Res 201:192-197. CrossRef Medline

Jeurissen B, Leemans A, Jones DK, Tournier JD, Sijbers J (2011) Probabilistic tractography using the residual bootstrap with constrained super-resolved spherical harmonic deconvolution. Hum Brain Mapp 32:461-479. CrossRef Medline

Jones DK, Horsfield MA, Simmons A (1999) Optimal strategies for measuring diffusion in anisotropic systems by magnetic resonance imaging. Magn Reson Med 42:515-525. CrossRef Medline

Jones DK, Catani M, Pierpaoli C, Reeves SJ, Shergill SS, O'Sullivan M, Maguire P, Horsfield MA, Simmons A, Williams SC, Howard RJ (2005) A diffusion tensor magnetic resonance imaging study of frontal cortex connections in very late-onset schizophrenia-like psychosis. Am J Geriat Psychiatry 13:1092-1099. CrossRef Medline

Jones DK, Knösche TR, Turner R (2013) White matter integrity, fiber count, and other fallacies: the do's and don'ts of diffusion MRI. Neuroimage 73:239-254. CrossRef Medline

Keedwell PA, Chapman R, Christiansen K, Richardson H, Evans J, Jones DK (2012) Cingulum white matter in young females at risk of depression: the effect of family history and anhedonia. Biol Psychiatry 72:296-302. CrossRef Medline

Kim S, Jeneson A, van der Horst AS, Frascino JC, Hopkins RO, Squire LR (2011) Memory, visual discrimination performance, and the human hippocampus. J Neurosci 31:2624-2629. CrossRef Medline

Lebel C, Beaulieu C (2011) Longitudinal development of human brain wiring continues from childhood into adulthood. J Neurosci 31:1093710947. CrossRef Medline

Lebel C, Walker L, Leemans A, Phillips L, Beaulieu C (2008) Microstruc- tural maturation of the human brain from childhood to adulthood. Neuroimage 40:1044-1055. CrossRef Medline

Lee ACH, Buckley MJ, Pegman SJ, Spiers H, Scahill VL, Gaffan D, Bussey TJ, Davies RR, Kapur N, Hodges JR, Graham KS (2005) Specialization in the medial temporal lobe for processing of objects and scenes. Hippocampus 15:782-797. CrossRef Medline

Leemans A, Jeurissen B, Sijbers J, Jones DK (2009) ExploreDTI: A graphical toolbox for processing, analyzing, and visualizing diffusion MRI data. In: 17th Annual Meeting of the International Society for Magnetic Resonance in Medicine, Honolulu, Hawaii, May 3-9.

Loui P, Li HC, Schlaug G (2011) White matter integrity in right hemisphere predicts pitch-related grammar learning. Neuroimage 55:500 507. CrossRef Medline

Metzler-Baddeley C, Jones DK, Belaroussi B, Aggleton JP, O’Sullivan MJ (2011) Frontotemporal connections in episodic memory and aging: a diffusion MRI tractography study. J Neurosci 31:13236-13245. CrossRef Medline

Metzler-Baddeley C, O'Sullivan MJ, Bells S, Pasternak O, Jones DK (2012) How and how not to correct for CSF-contamination in diffusion MRI. Neuroimage 59:1394-1403. CrossRef Medline

Mundy ME, Downing PE, Dwyer DM, Honey RC, Graham KS (2013) A critical role for the hippocampus and perirhinal cortex in perceptual learning of scenes and faces: complementary findings from amnesia and fMRI. J Neurosci 33:10490-10502. CrossRef Medline

Pasternak O, Sochen N, Gur Y, Intrator N, Assaf Y (2009) Free water elimination and mapping from diffusion MRI. Magnet Reson Med 62:717730. CrossRef Medline

Rochefort C, Lefort JM, Rondi-Reig L (2013) The cerebellum: a new key structure in the navigation system. Front Neural Circuits 7:35. CrossRef Medline

Rudebeck SR, Scholz J, Millington R, Rohenkohl G, Johansen-Berg H, Lee ACH (2009) Fornix microstructure correlates with recollection but not familiarity memory. J Neurosci 29:14987-14992. CrossRef Medline

Saksida LM, Bussey TJ (2010) The representational-hierarchical view of amnesia: Translation from animal to human. Neuropsychologia 48: 2370-2384. CrossRef Medline

Sang L, Qin W, Liu Y, Han W, Zhang Y, Jiang T, Yu C (2012) Resting-state functional connectivity of the vermal and hemispheric subregions of the cerebellum with both the cerebral cortical networks and subcortical structures. Neuroimage 61:1213-1225. CrossRef Medline

Saunders RC, Aggleton JP (2007) Origin and topography of fibers contributing to the fornix in macaque monkeys. Hippocampus 17:396-411. CrossRef Medline

Saygin ZM, Norton ES, Osher DE, Beach SD, Cyr AB, Ozernov-Palchik O, Yendiki A, Fischl B, Gaab N, Gabrieli JDE (2013) Tracking the roots of reading ability: white matter volume and integrity correlate with phonological awareness in prereading and early-reading kindergarten children. J Neurosci 33:13251-13258. CrossRef Medline

Smith SM, Nichols TE (2009) Threshold-free cluster enhancement: addressing problems of smoothing, threshold dependence and localisation in cluster inference. Neuroimage 44:83-98. CrossRef Medline

Smith SM, Jenkinson M, Johansen-Berg H, Rueckert D, Nichols TE, Mackay CE, Watkins KE, Ciccarelli O, Cader MZ, Matthews PM, Behrens TE (2006) Tract-based spatial statistics: voxelwise analysis of multi-subject diffusion data. Neuroimage 31:1487-1505. CrossRef Medline

Thomas C, Avidan G, Humphreys K, Jung KJ, Gao F, Behrmann M (2009) Reduced structural connectivity in ventral visual cortex in congenital prosopagnosia. Nat Neurosci 12:29-31. CrossRef Medline

Tournier JD, Yeh CH, Calamante F, Cho KH, Connelly A, Lin CP (2008) Resolving crossing fibres using constrained spherical deconvolution: validation using diffusion-weighted imaging phantom data. Neuroimage 42:617-625. CrossRef Medline

Vann SD, Denby C, Love S, Montaldi D, Renowden S, Coakham HB (2008) Memory loss resulting from fornix and septal damage: impaired supraspan recall but preserved recognition over a 24-hour delay. Neuropsychology 22:658-668. CrossRef Medline

Wakana S, Caprihan A, Panzenboeck MM, Fallon JH, Perry M, Gollub RL, Hua K, Zhang J, Jiang H, Dubey P, Blitz A, van Zijl P, Mori S (2007) Reproducibility of quantitative tractography methods applied to cerebral white matter. Neuroimage 36:630-644. CrossRef Medline

Wilson CRE, Charles DP, Buckley MJ, Gaffan D (2007) Fornix transection impairs learning of randomly changing object discriminations. J Neurosci 27:12868-12873. CrossRef Medline 